\title{
Skin Colour Analysis of Iraqi Kurdish Population
}

\author{
Faraedon M. Zardawi, PhD. Dept of Periodontology, School of Dentistry, University of Sulaimani, Sulemani, Iraq. \\ Corresponding Author, faraedon.mostafa@univsul.edu.iq, 009647702263062 \\ Kaida Xiao, PhD. Academic Unit of Adult Dental Care, School of Clinical Dentistry, University of Sheffield, Clare- \\ mont Crescent, Sheffield S10 2TA, UK. \\ Julian M. Yates, PhD. Dept of Oral \& Maxillofacial Surgery, School of Dentistry, University of Manchester, Coup- \\ land III Building, Coupland Street M13 9PL, UK.
}

\begin{abstract}
Objective:

Skin colour measurement and analysis was performed for Iraqi Kurdish population in sulaimani city. The purpose of this study was to produce a dedicated skin shade guide for precise colour reproduction and colour matching of maxillofacial prostheses with the patient's original skin colour.
\end{abstract}

\section{Methodology:}

A skin colour measurement was undertaken for 140 subjects ( 73 female and 67 male). A method of capturing their $\left(L^{*} a^{*} b^{*}\right)$ colour values from nine body parts was performed using a Minolta CM-2600d spectrophotometer and a colour Data Software. The study sample aged between 18 to 50 years from Sulaimani city representing a Kurdish population of the north of Iraq. A comparison was made between $\left(L^{*} a^{*} b^{*}\right)$ colour values of the face and arm and between male and female shades. Data analysed using Independent-samples T-Test. Furthermore this study aimed at employing the performed data for suggesting a dedicated skin shade guide for the population examined in this study.

\section{Results:}

A significant difference between facial and arm colour was identified. Facial colour found to be more reddish and colourful but darker than arm colour. Furthermore, collected data showed lighter female colour than males. Variations in skin colour of the study population were detected, body area differences and gender differences found to have a significant effect on the skin colour formulation of the study population. Based on colour data obtained, a skin shade guide was suggested for the population examined in this study.

Conclusions:

A consistent colour shift in Kurdish population between facial and arm colour in which facial colour found to be reddish, more colourful, and darker than arm colour. Females are found to have a lighter colour than males.

A dedicated skin shade guide was developed and suggested for Kurdish population based on a comprehensive colour gamut that was determined in this study.

\section{Skin Shade Guide, (L*A*B*) Colour Values, Maxillofacial Prostheses, Colour Gamut}

\section{Introduction}

Colour matching and colour differences between the maxillofacial prostheses and the surrounding skin is a prime concern of the maxillofacial prosthodontist and the patient. Failure of achieving the patient's exact skin colour lead to aesthetic failure (Lontz, 1990). Colour discrepancy is extremely evident in the maxillofacial region especially when the colour of the prosthesis is not accurately blended into the patient's skin colour. Thus, a properly fitted prosthesis and anatomically accurate prosthesis will be refused by the patient if its colour is not appropriately matching the adjoining tissues (Guttal et al., 2008). Skin colour varies according to various locations in the body, these differences may create aesthetic problems for the patient after micro-vascular surgery (Shaw and Ahn, 1992, Feldman, 1990). The desired results may not be achieved as a result of site differences of the transplanted tissue.

Several factors affect the human skin colour, include, the thickness of epidermis layer and the amount of blood supply. Variation in natural skin color is mainly due to genetic factors (Walters and Roberts, 2008). However, the greatest source of colour in human skin is the types and amount of melanin pigments, which is responsible for colour variation among different ethnic groups (Thong et al., 2003, Ito and Wakamatsu, 2003). 
Human skin colour also influenced by exposure to sunlight that cause tanning of skin. The exposed parts of the skin exhibit darker colour than nonexposed skin. Today, maxillofacial technicians depend on visual assessment which is based on visual acuity for reproducing the patient's colour (Polyzois, 1999, Seelaus R and RJ, 2000).

Digital colour capturing devices (Camera, spectrophotometer, and colorimeter) identify and present colour in 3 dimensions by using 3D colour models (Coward et al., 2008a, Hunter, 1987). Today, RGB colour model and CIE LAB are the most widely used colour models. Colorimeter and spectrophotometer are used to improve the accuracy of colour matching by measuring the skin colour numerically in three dimensions (Koran et al., 1981).

In order to measure objectively the skin colour appearance and evaluate skin colour differences, CIE Colorimetry has been widely used for specifying skin colours into CIELAB uniform colour space.

Human skin tone is confined to a limited zone within the whole colour space, which is referred to as skin colour gamut (Stone et al., 1988). Recognition of colour gamut of human skin provides clear information about the distribution/location of the colour value among the whole colour space. Knowing the colour gamut of a population makes developing a shade guide for that population. To date, no comprehensive global database for skin colour among the main ethnic groups is available. Generation of a global skin shade guide requires conducting a comprehensive measurement of skin colour among groups of people from different ethnic backgrounds and different geographic locations around the world. Skin colour measurements have seldom been conducted among individual ethnic groups (Over et al., 1998, Coward et al., 2008b, Xiao et al., 2012, Han et al., 2006) and some of these were performed only for limited purposes. On searching databases, no published articles were found providing colour information for the Kurdish population in the Kurdistan region. Therefore, the purpose of the present study was to conduct colour measurement procedure and to assemble colour information from a group of Kurdish population from Sulaimani city - Iraq. Moreover, the current study was conducted to determine the skin colour range of Kurdish population and to suggest data for developing a dedicated shade guide for their skin colour.

\section{Methodology}

1-Ethical approval has been obtained from the University Research Ethics Committee (School of Dentistry - University of Sulaimani) for measuring skin colours for a group of volunteers included students and the school employees. An information sheet, including all information and details about the colour measurement procedure has been produced for the volunteers. A consent form to be read and signed by the volunteer was also produced.

2-140 Volunteers from the School of Clinical Dentistry - University of Sulaimani, 73 female and 67 male were participated in this study. The participants included staff and students of the dental school within an age group 18-50 years. A total of $1260(140 \times 9)$ skin colour data was collected. For each subject, skin colour of a total of nine body parts obtained, forehead, tip of nose, cheek, ear lobe, chin, back of hand, palm, forearm outer and forearm inter (Figure 1). Moreover, each subject also provided his basic information including age and gender.

3-A Konica Minolta CM-2600d spectrophotometer using SpectraMagic NX Colour Data Software was employed to measure the skin colour in CIELAB tristimulus values with a
2 degree standard observer. Colour instruments (colormetry) generate tristimulus values in an $X, Y$ and $Z$ coordinate system. Depending on the specific instrument, these tristimulus values are converted to Hunter $L, a, b$ or CIELAB $L^{*}, a^{*}$ and $b^{*}$ units. The difference between the two systems is the mathematical manipulation of the basic tristimulus values (PolyOne, 2005)The illuminate was set to the CIE standard D65 to simulate skin colour in daylight conditions. During the measurement, a viewing geometry of $d / 8$ (diffuse illumination, 8-degree viewing), was used by the specular component included and the aperture size was set to $3 \mathrm{~mm}$.

4-Independent-samples T-Test was conducted to present the results and to analyse the differences of the three colour attributes $\left(L^{*}, a^{*}\right.$ and $\left.b^{*}\right)$. The $L^{*} a^{*} b^{*}$ is described as the vertical $\left(L^{*}\right)$ axis which represent lightness, ranging from 0-100 and two other horizontal axis values ( $\left.a^{*}\right)$ and $\left(b^{*}\right)$. Each has an "axis" that can range from $(-a)$ to $(+a)$ and $(-b)$ to $(+b)$. More specifically, $(-a)$ represents green and $(+a)$ represents red, whilst $(b+)$ is represented by yellow and (b-) is represented by blue, as is shown in Figure 2 (Hunter, 1948, Honiball, 2010).

5 -Based on colour data obtained in this study a suggestion was made for developing a skin shade guide for the study population as follow: Ten selected shades were used to develop a dedicated shade guide based on the higher and lower measurements detected for each of the three colour attributes $\left(L^{*}, a^{*}\right.$ and $\left.b^{*}\right)$ among 1260 measurements - the total $\left(L^{*} a^{*} b^{*}\right)$ measurements. The process of selection carried out by taking one measurement below the lower margin, and then graduating to a higher level for each shade, the graduation continued up to shade number 10 , this was one measurement above the higher margin of the total records for each colour attribute. 
Results:

Table 1 presents the average and standard deviation of $\left(L^{*}, a^{*}\right.$ and $\left.b^{*}\right)$ values of skin tone and the higher and the lower skin tone range for each colour attribute of the total sample. An average $\left(L^{*}, a^{*}\right.$ and $\left.b^{*}\right)$ values reported $(56.4,10.2$ and 15.8$)$ respectively.

Table 1 also details the $\left(L^{*} a^{*} b^{*}\right)$ colour distribution according to gender and body parts. It can be seen that females have significantly $(p<0.05)$ lighter skin shades as 2 tailed independent samples t-test showed.

A great variation in $\left(L^{*}\right)$ values (lightness) between male and female, statistically a significant difference $(p<0.05)$ of $\left(L^{*}\right)$ Value were recorded between male and female (59 against $54.3)$ respectively. A significant difference $(p<0.05)$ of $\left(a^{*}\right)$ Value was also recorded between male and female (9.9 against 10.5) respectively, whereas, no significant differences $(p>0.05)$ was reported among the sample of $\left(b^{*}\right)$ values between male and female (15.6 to 15.9) respectively. However, males showed slightly more colorful skin than female and this is represented by higher $\left(a^{*}\right)$ values for males against female. Differences in the lightness, $\left(L^{*}\right)$ values were the greatest between female and male's chins, (59) against (49.2) respectively.

Figure 3 presents the average and standard error for $\left(L^{*}, a^{*}\right.$ and $\left.b^{*}\right)$ values differences between face and arm among the total sample. In general, the results demonstrate a clear trend that face is significantly $(p<0.05)$ darker and reddish than arm. This is presented with a significantly lower $\left(\mathrm{L}^{*}\right)$ and a higher $\left(\mathrm{a}^{*}\right)$ values (55.2, 11.9) of the face against the arm (58.4, 8.1) respectively. However, there were no significant differences $(p>0.05)$ in the $\left(b^{*}\right)$ values between face and arm shades (15.9 against 15.7) respectively.

Variations of $\left(L^{*} a^{*} b^{*}\right)$ colour attri-

\begin{tabular}{|c|c|c|c|c|c|c|}
\hline \multirow{4}{*}{ Total } & \multicolumn{6}{|c|}{ L*a*b* Values Total sample } \\
\hline & \multicolumn{2}{|c|}{ Values } & $\mathrm{L}^{*}$ & a* & \multicolumn{2}{|c|}{$b^{*}$} \\
\hline & \multicolumn{2}{|c|}{ Average } & 56.4 & 10.2 & \multicolumn{2}{|c|}{15.8} \\
\hline & \multicolumn{2}{|c|}{ Min - Max } & $32-70.7$ & $\begin{array}{c}3.8- \\
19.1\end{array}$ & \multicolumn{2}{|c|}{$7.5-22$} \\
\hline Gender & \multicolumn{3}{|c|}{ Female - 73 Subjects } & \multicolumn{3}{|c|}{ Male -67 Subjects } \\
\hline Body Position & $\mathrm{L}^{*}$ & a* & $b^{*}$ & $\mathrm{~L}^{*}$ & a* & $b *$ \\
\hline Face & \multicolumn{6}{|c|}{ FACE - L*a*b* Values } \\
\hline Forehead & 57.2 & 10.4 & 15.7 & 54.8 & 12.2 & 17.1 \\
\hline Nose & 57.9 & 13.2 & 15.9 & 54.5 & 14 & 16.1 \\
\hline Cheek & 60.4 & 11.7 & 15.6 & 55.6 & 11.7 & 16.1 \\
\hline Ear & 51.8 & 10.4 & 15.2 & 51.6 & 12.3 & 15.7 \\
\hline Chin & 59 & 13.5 & 15.9 & 49.2 & 9 & 13.1 \\
\hline Av\&Sd & $57.3 \pm 3.2$ & $11.8 \pm 1.5$ & $15.7 \pm 0.3$ & $53.1 \pm 2.7$ & $11.8 \pm 1.8$ & $15.6 \pm 1.5$ \\
\hline Arm & \multicolumn{6}{|c|}{ ARM - L*a*b* Values } \\
\hline Backhand & 60 & 7.9 & 16.6 & 55 & 9.2 & 16.3 \\
\hline Palm & 61.4 & 9.1 & 14 & 59 & 10.7 & 14.1 \\
\hline Outer Arm & 59.9 & 6.8 & 16.5 & 50.9 & 7.8 & 17.6 \\
\hline Inner Arm & 63.4 & 5.7 & 15.4 & 57.9 & 7.4 & 17.3 \\
\hline Av\&Sd & $61.2 \pm 1.6$ & $7.4 \pm 1.5$ & $15.6 \pm 1.2$ & $55.7 \pm 3.6$ & $8.8 \pm 1.5$ & $16.3 \pm 1.6$ \\
\hline Total-AV\&SD & $59 \pm 3.3$ & $9.9 \pm 2.7$ & $15.6 \pm 0.8$ & $54.3 \pm 3.2$ & $10.5 \pm 2.3$ & $15.9 \pm 1.5$ \\
\hline
\end{tabular}

Table 1: Average L*a*b* Values - Total average and upper and lower range values of $L^{*} a * b *$ for different body parts according to gender

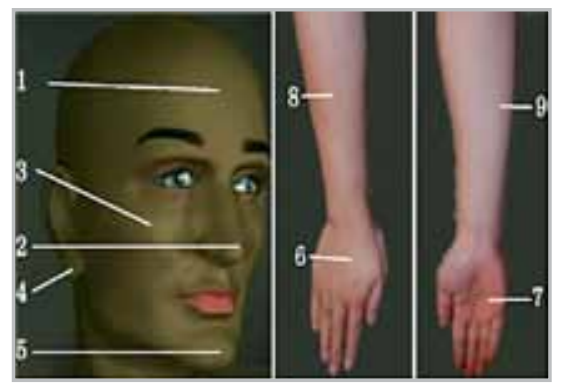

Figure 1: position for skin measurements

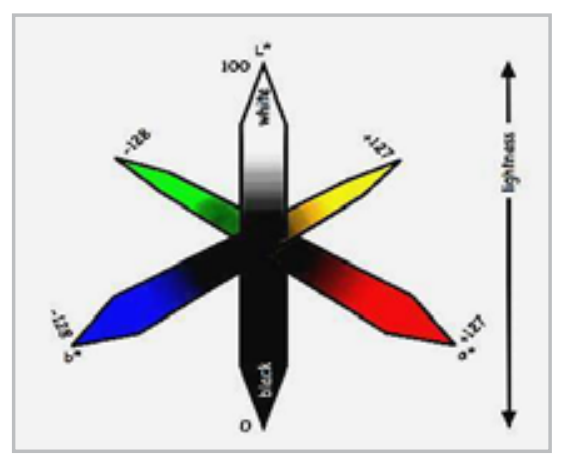

Figure 2: L*a*b* colour model butes in the 'colour gamut' among the sample is presented in Table 1 and Figure 4. The average value for each attribute $\left(L^{*}\right),\left(a^{*}\right)$ and $\left(b^{*}\right)$ and the highest and lowest values for these components were marked in order to determine the range of colour distribution for the sample. There was a great variation in $\left(L^{*}\right)$ value, which ranged from (32) to (70.7) with a mean value of (56.4). This indicates that the skin of the Kurdish population is a combination of light and dark skin tones. Figure 5 presents the suggested $\left(L^{*} a^{*} b^{*}\right)$ values for $a$ skin shade guide for Kurdish population which was developed according to the protocol mention in the previous section. Colour attributes of these 10 shades $\left(L^{*}, a^{*}\right.$ and $\left.b^{*}\right)$ followed $a$ linear distribution on a plotted histogram. This collection can be suggested as a dedicated skin shade guide for the study sample. 


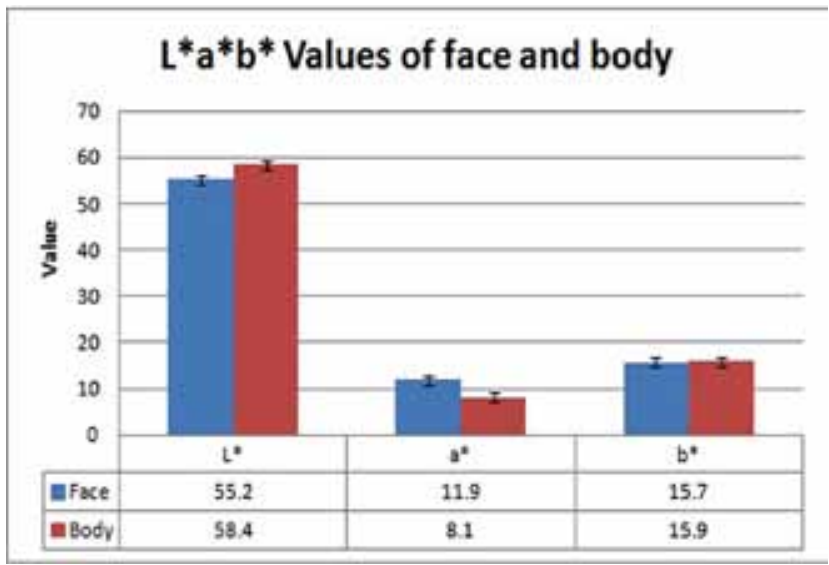

Figure 3: $L * a * b *$ differences between face and arm

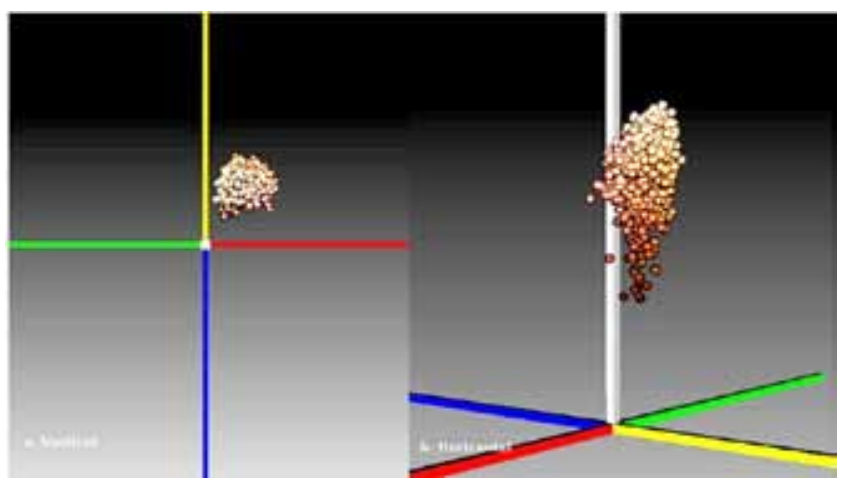

Figure 4: Vertical (a) and horizontal (b) appearance of the skin colour gamut for the sample

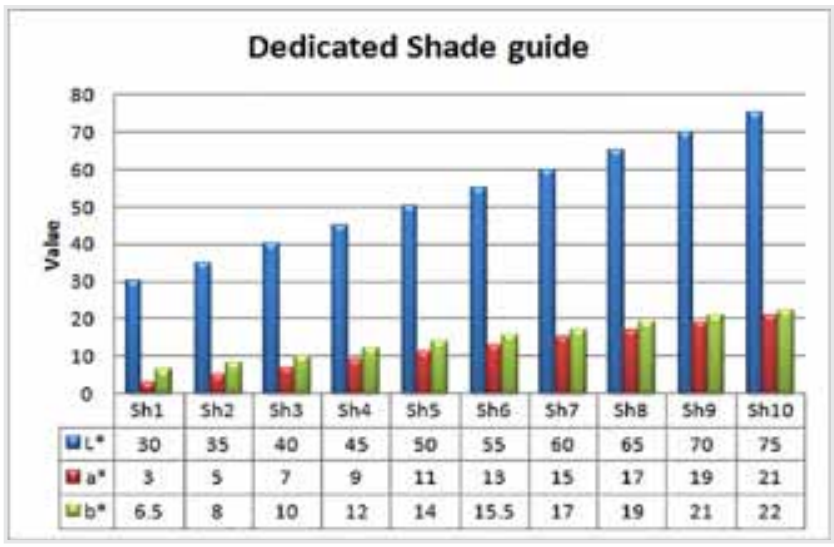

Figure 5: Distribution of $L^{*}, a^{*}$ and $b^{*}$ attributes in the suggested shade guide

\section{Discussion:}

Human skin colour shows variations throughout life, and human skin undergoes several structural and functional alterations caused by intrinsic aging and independent environmental insults (Roh et al., 2001). This often results in hypo pigmentation or hyper pigmentation spots by erratic melanocyte activity as a result of cumulative UV expo- sure. Structurally, the aged epidermis likely becomes thinner and less coloured than the young epidermis. With age, human skin loses colour and presents a significant reduced in $\left(a^{*}\right)$ and $\left(b^{*}\right)$ values (Fullerton and Serup, 1997). In order to collect more valid clear and representative skin colour data, the majority of data was collected from a younger age group - mainly students aged from 20-25 years.

The current study aimed to formulate a comprehensive skin colour database and skin colour information based on CIELAB tristimulus values for males and females volunteers.

Skin colour gamut for the sample, and colour variations according to gender and body part were also determined. The results provided some important evidence about the skin colour of the population investigated in this study. This evidence suggested that Kurdish shad is a combination of dark and light tones. Furthermore, females exhibited lighter skin shade than males, while males exhibited redder skin colour than females: the redness can be attributed to the greater blood volume in the male's body (Fortney et al., 1981). This study result agree with a previous study performed to collect Skin colour data onto the population of Nowshahr City, northern Iran, which revealed significant differences between the males and females on the upper inner arm and the forehead, and within each sex between the sites (Mehrai and Sunderland, 1990). However, the lightness is probably due to genetic factors, such as the male having more melanin in his skin or the fact that most outdoor labor is undertaken by males. Also, females are more likely to use makeup, which provides some protection from sunlight (Brown et al., 1962, Frost, 2006). The data also revealed that faces are of darker and more colorful shades than the arms. This is probably due to the fact that the face is an uncovered part of the body and exposed to more sunlight than the covered parts. The natural skin colour can darken as a result of tanning due to exposure to sunlight. Either the UV-radiation creates oxidative stress, which in turn oxidizes existing melanin and leads to rapid darkening of the melanin or there is an increase in production of melanin to provide protection to the skin (Agar and Young, 2005). Differences in the lightness, $\left(L^{*}\right)$ values were the greatest between female and male's chins (59 against 49.2 ) respectively, this is probably because of thick subcutaneous hair follicles on some male's chin as the table shows.

It is very important to consider these facts when applying data acquisition or manufacturing processes to skin colour reproduction. Human skin shades can be digitally represented using cameras (2D or 3D) and then physically reproduced using manual - handmade or digital - additive manufacturing techniques. Layered fabrication techniques such as 3D colour printing is recently adopted for fabrica- 
tion of Soft tissue facial prostheses (Zardawi, 2013). For this process to be successful, the appropriate skin colour gamut has to be used as a true reference in order to achieve an accurate skin colour reproduction and/ or translation between different media.

Another important advantage of skin colour measurement is to provide each population/ethnic group with a dedicated skin shade guide instead of one shade guide representing the entire skin colour range. If, for example, one wants to produce a prosthesis for a patient in China, all that is needed is to refer to the Chinese skin shade guide. This will allow much more focused and more accurate colour matching. One skin shade guide for the entire population of the world cannot be as accurate as a range of dedicated skin shade guides. If we look at any ethnic shade, for example, it is possible to produce a skin shade guide for an ethnic group, perhaps comprising no more than eight to ten skin shades. This could not accurately fit the selected population properly, there might be some individual variations, not all individuals fell in the selected colour range dedicated for a specific population, some individuals might have a different skin colour fell out of the shade guide's range. However, the closest colour match could be achieved and modified to fit the required shade properly. Moreover, the suggested skin shade guide should be produced and its validity and accuracy should be tested on human skin in order to perform the required improvement to achieve a more inclusive skin shade guide for the selected population. This kind of a very simple shade guide can be created for the purpose of producing prosthetic devices matching the patient's skin colour properly.

\section{Conclusions}

Consistent colour shift in Kurdish population between facial and arm colour in which facial colour found to be reddish, more colourful, and darker than arm colour. Females are found to have a lighter colour than males.

A comprehensive colour gamut was developed and a dedicated skin shade guide for the study population, which requires further testing and clinical validation.

\section{References}

AGAR, N. \& YOUNG, A. R. 2005. Melanogenesis: a photoprotective response to DNA damage? Mutation Research/Fundamental and Molecular Mechanisms of Mutagenesis, 571, 121-132.

BROWN, E., HOPPER JR, J., HODGES JR, J., BRADLEY, B., WENNESLAND, R. \& YAMAUCHI, H. 1962. Red cell, plasma, and blood volume in healthy women measured by radiochromium cell-labeling and hematocrit. Journal of Clinical Investigation, 41, 2182.

COWARD, T., SEELAUS, R. \& LI, S. 2008a. Computerized Color Formulation for African $\nabla$ Canadian People Requiring Facial Prostheses: A Pilot Study. Journal of Prosthodontics, 17, 327-335.

COWARD, T. J., SEELAUS, R. \& LI, S. Y. 2008b. Computerized color formulation for African-Canadian people requiring facial prostheses: a pilot study. J Prosthodont, 17, 327-35. FELDMAN, J. F. B. I. M., JG. (ED.) 1990. Plastic Surgery. Philadelphia: WB Saunders, 3, 2153 - 2236.

FORTNEY, S., NADEL, E., WENGER, C. \& BOVE, J. 1981. Effect of blood volume on sweating rate and body fluids in exercising humans. Journal of Applied Physiology, 51, 1594-1600.

FROST, P. 2006. European hair and eye color: A case of frequency-dependent sexual selection? Evolution and Human Behavior, 27, 85-103.

FULLERTON, A. \& SERUP, J. 1997. Site, gender and age variation in normal skin colour on the back and the forearm: tristimulus colorimeter measurements. Skin Research and Technology, 3, 49-52.

GUTTAL, S. S., PATIL, N. P., NADIGER, R. K. \& KULKARNI, R. 2008. A study on reproducing silicone shade guide for maxillofacial prostheses matching Indian skin color. Indian J Dent Res, 19, 191-5.

HAN, K., CHOI, T. \& SON, D. 2006. Skin color of Koreans: statistical evaluation of affecting factors. Skin Research and Technology, 12, 170177.

HONIBALL, J. R. 2010. The Application of 3D Printing in reconstructive surgery. MSc Theses, University of Stellenbosch

HUNTER, R. R. S., HAROLS RW. 1987. The Measurement of Appearance, PP. pp, ed 2, ed 2, pp 7-13.

HUNTER, R. S. 1948. Proceedings of the Winter Meeting of the Optical Society of America. JOSA, Jornal of Optical Sociaty of America, 38 (7), 661.

ITO, S. \& WAKAMATSU, K. 2003. Quantitative analysis of eumelanin and pheomelanin in humans, mice, and other animals: a comparative review. Pigment Cell Res, 16, 523-31. KORAN, A., POWERS, J. M., RAPTIS, C. N. \& YU, R. 1981. Reflection spectrophotometry of facial skin. J Dent Res, 60, 979-82.

LONTZ, J. F. 1990. State-of-theart materials used for maxillofacial prosthetic reconstruction. Dent Clin North Am, 34, 307-25.

MEHRAI, H. \& SUNDERLAND, E. 1990. Skin colour data from Nowshahr City, northern Iran. Annals of human biology, 17, 115-120.

OVER, L. M., ANDRES, C. J., MOORE, B. K., GOODACRE, C. J. \& MUNOZ, C. A. 1998. Using a colorimeter to develop an intrinsic silicone shade guide for facial prostheses. J Prosthodont, 7, 237-49.

POLYONE 2005. Interpretation of Color Data (A Simplistic Overview). www.Poly0ne.com, 79.

POLYZOIS, G. L. 1999. Color stabil- 
ity of facial silicone prosthetic polymers after outdoor weathering. J Prosthet Dent, 82, 447-50.

ROH, K. Y., KIM, D., HA, S. J., RO, Y. J., KIM, J. W. \& LEE, H. J. 2001. Pigmentation in Koreans: study of the differences from Caucasians in age, gender and seasonal variations. British Journal of Dermatology, 144, 94-99.

SEELAUS R \& RJ, T. 2000. . Facial prosthesis fabrication: coloration techniques. In: Clinical maxillofacial prosthetics. . TaylorTD, editor. Chicago, IL: Quintessence Publishing, chapter 17, 245-264.

SHAW, W. W. \& AHN, C. Y. 1992. Microvascular free flaps in breast reconstruction. Clin Plast Surg, 19, 917-26.

STONE, M. C., COWAN, W. B. \& BEATTY, J. C. 1988. Color gamut mapping and the printing of digital color images. ACM Transactions on Graphics (TOG), 7, 249-292.

THONG, H. Y., JEE, S. H., SUN, C. C. \& BOISSY, R. E. 2003. The patterns of melanosome distribution in keratinocytes of human skin as one determining factor of skin colour. $\mathrm{Br}$ J Dermatol, 149, 498-505.

WALTERS, K. A. \& ROBERTS, M. S. 2008. Dermatologic, cosmeceutic, and cosmetic development: Therapeutic and novel approaches. New York: Informa Healthcare.

XIA0, K., LIAO, N., ZARDAWI, F., LIU, H., NOORT, R. V., YANG, Z., HUANG, M. \& YATES, J. M. 2012. Investigation of Chinese skin colour and appearance for skin colour reproduction. Chinese Optics Letters, 10, 083301.

ZARDAWI, F. M. 2013. Characterisation of Implant Supported Soft Tissue Prostheses Produced with 3D Colour Printing Technology. 